\title{
The organization of stable pre-crystallization phase from oligomers in lysozyme solution with different precipitates and the thin films formation at the air-liquid/air-substrate interfaces from the crystallization solutions
}

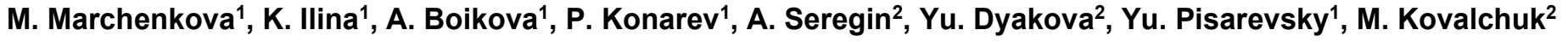 \\ ${ }^{I}$ Shubnikov Institute of Crystallography of Federal Scientific Research Centre "Crystallography and Photonics" of Russian Academy \\ of Sciences, Leninskii pr. 59, Moscow, 119333, Russian Federation, \\ ${ }^{2}$ National Research Centre "Kurchatov Institute”, Akademika Kurchatova pl. 1, Moscow, 123182, Russian Federation
}

marchenkova@crys.ras.ru

Protein crystallization is a key step in enabling structural studies using crystallographic methods. Despite much research and experimental progress in this area, there is still no clear model to explain the mechanisms and physicochemical principles for all of the stages of crystallization. Studying the mechanisms of crystallization at the earliest possible stage of the procedure, including rapid characterization of the solution and protein solubility, would greatly enhance the prediction of potential crystal formation.

The process of the protein cluster formation was discussed and the attempts to observe it were made, but the structure of the formed clusters remained unknown. Recently, we have proposed the hypothesis that there is a pre-crystallization phase in solution composed of protein oligomers, where these oligomers are the elements of the resulting crystal structure. On the base of crystal structure of tetragonal lysozyme crystals octomer cluster was selected as a possible element of crystal growth [1]. These octamers were found in crystallization solutions by small-angle X-ray and neutron scattering methods (SAXS and SANS). The results show noticeable presence of lysozyme dimers and octamers under crystal growth conditions and total absense of oligomers under conditions when crystal growth is impossible. The influence of the precipitant cation type in a series of chlorides $\left(\mathrm{NaCl}, \mathrm{KCl}, \mathrm{LiCl}_{1} \mathrm{NiCl}_{2}, \mathrm{CuCl}_{2}\right.$, $\mathrm{CoCl}_{2}$ ) on the structure of lysozyme solutions was also investigated [2]. The bonds between lysozyme molecules and precipitant ions in single crystals grown with chlorides of these metals are analysed based on crystal structure data [3].

The relationship between various parameters of crystallization solutions and the possibility of protein oligomers formation under selected conditions could be useful for ordered films formation. The formation of the Langmuir films from crystallization solutions were studied by grazing-incidence X-ray standing waves [4]. Studying the formation of these monolayers by GIXSW showed that the thickness of the resulting protein layer was twice the diameter of an individual lysozyme molecule and matched the diameter of the octamer. Thin layers of precipitant ions $(\mathrm{K}$ and $\mathrm{Cl})$ formed directly under the protein monolayer.
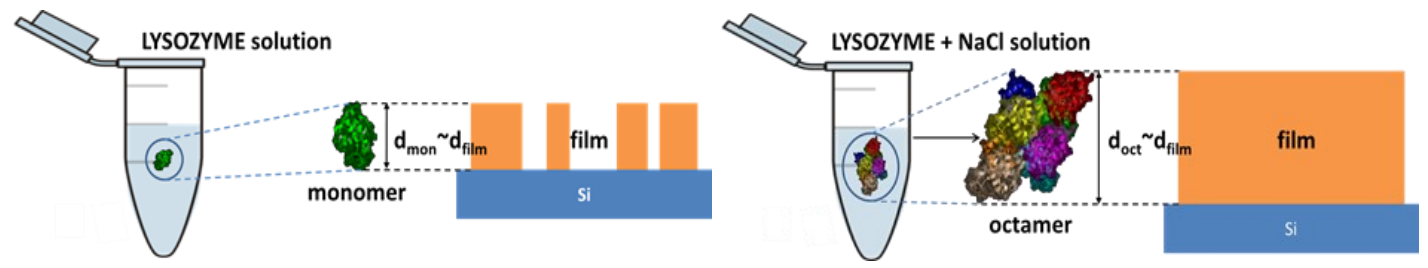

Figure 1. The proposed structure of LB lysozyme films dependent on the initial lysozyme solution: a) solution of lysozyme monomers lead to $3 \mathrm{~nm}$ thickness film organization with rare islands; b) solution of octamers (which are formed under crystallization condition with $\mathrm{NaCl}$ precipitant) leads to uniform film formation.

[1] Kovalchuk M. V., Blagov A.E., Dyakova Y.A., Gruzinov A.Y., Marchenkova M.A., Peters G.S., Pisarevsky Y. V., Timofeev V.I., Volkov V. V. (2016). Crystal Growth \& Design 16 (4), 1792.

[2] Dyakova Y.A., Boikova A.S., Ilina K.B., Konarev P. V., Marchenkova M.A., Pisarevsky Y. V., Timofeev V.I., Kovalchuk M. V. (2019). Crystallography Reports 64 (1), 11.

[3] Marchenkova M.A., Kuranova I.P., Timofeev V.I., Boikova A.S., Dorovatovskii P. V., Dyakova Y.A., Ilina K.B., Pisarevskiy Y. V., Kovalchuk M. V. (2019). Journal of Biomolecular Structure and Dynamics in press, 1.

[4] Kovalchuk M. V., Boikova A.S., Dyakova Y.A., Ilina K.B., Konarev P. V., Marchenkova M.A., Pisarevskiy Y. V., Prosekov P.A., Rogachev A. V., Seregin A.Y. (2019). Thin Solid Films 677 (February), 13.

Keywords: Protein Oligomers, Protein solution, Protein Crystallization, Crystal Growth, SAXS, SANS, Langmuir films, XSW

This study was supported in part by the Ministry of Science and Higher Education within the State assignment FSRC "Crystallography and Photonics» RAS and by the Russian Foundation for Basic Research (project number 19-29-12042 mk).

Acta Cryst. (2021), A77, C1225 